\title{
A cutoff point for arterial stiffness using the cardio-ankle vascular index based on carotid arteriosclerosis
}

\author{
Huaqing $\mathrm{Hu}^{1,6}$, Huan $\mathrm{Cui}^{2,6}$, Weixing $\mathrm{Han}^{3}$, Liangping $\mathrm{Ye}^{1}$, Wenting Qiu ${ }^{4}$, Hui Yang ${ }^{4}$, Chuanwu Zhang ${ }^{4}$, \\ Xiaojuan $\mathrm{Guo}^{4,5}$ and Guangyun Mao ${ }^{4}$
}

The cardio-ankle vascular index (CAVI) has been widely accepted as a good indicator of arteriosclerosis. However, the lack of a reliable diagnostic criterion for CAVI hampers the proper clinical screening for arteriosclerosis using CAVI and impedes the prompt treatment of cardiovascular disease (CVD). There is an urgent need to determine a criterion for CAVI in arteriosclerosis prevention. We conducted a cross-sectional study to determine this criterion based on receiver operating characteristic (ROC) analyses in a Chinese population consisting of 328 participants. CAVI was measured in duplicate, and carotid ultrasound detection was performed in a quiet environment by well-trained physicians. After multivariate adjustment, CAVI was positively associated with the risk of carotid arteriosclerosis. Compared with participants in the lowest tertile of CAVI (5.15-7.40), those in the medium (7.41-8.65) and highest (8.66-13.60) tertiles had odds ratios $(95 \%$ confidence interval) of $2.2(1.0,4.9)$ and $4.4(1.5,13.3)$, respectively, for developing carotid arteriosclerosis $(P$ trend $=0.007)$. The areas under the ROC curve (AUC) of the male, female and pooled populations were $0.789,0.897$ and 0.856 , respectively. The cutoff point of CAVI $\geqslant 8.0$ resulted in the largest sensitivity and specificity. Furthermore, CAVI and age acted synergistically to increase the risk of carotid arteriosclerosis. CAVI $\geqslant 8.0$ may be an optimal cutoff point for carotid arteriosclerosis prediction. The older population with higher CAVI scores had a higher risk of carotid arteriosclerosis. Additional large prospective studies are needed to confirm our findings.

Hypertension Research (2013) 36, 334-341; doi:10.1038/hr.2012.192; published online 17 January 2013

Keywords: cardio-ankle vascular index; carotid arteriosclerosis; cardiovascular disease; cross-sectional study; synergistic effect

\section{INTRODUCTION}

Cardiovascular disease (CVD) is the leading cause of morbidity and mortality in developed countries. ${ }^{1}$ Additionally, CVD has emerged as the major health challenge in China as the lifestyles and dietary habits of Chinese people have greatly changed over the past few decades. According to a WHO estimate, of the 29 million deaths caused by non-communicable diseases ( $\sim 51 \%$ of the 57 million deaths from all causes in 2008), $59 \%$ were caused by CVD, followed by $26 \%$ by cancer and $15 \%$ by respiratory diseases. ${ }^{2}$ Many studies have been performed to explore the association between CVD and other factors. Current evidence has confirmed that arteriosclerosis is one of the most important contributors to the development of CVD. ${ }^{1,2}$ It is necessary to identify persons with moderate-to-advanced states of arteriosclerosis as early as possible. As previous studies have demonstrated, the stiffening of the arterial wall is one of the most important mechanisms for developing CVD, with the degree of arteriosclerosis being a good surrogate marker for determining the prognosis of CVD. ${ }^{3,4}$ In the past few decades, the assessment of arterial stiffness has become a widely used tool for investigating the function of arteries. Traditionally, pulse wave velocity (PWV) has been widely used to quantitatively estimate the state of arteriosclerosis. PWV is a non-invasive parameter for predicting cardiovascular mortality and has been shown to be significantly affected by many factors, such as age, blood pressure (BP), hyperglycemia, dyslipidemia, cigarette smoking and alcohol consumption, among Japanese Americans. ${ }^{5}$ To overcome the limitations of PWV, the cardio-ankle vascular index (CAVI) was developed in 2004 as a new indicator of arteriosclerosis to quantitatively estimate the extent of arterial stiffness. ${ }^{6}$ Because its measurement is not significantly influenced by changes in BP, CAVI

${ }^{1}$ Physical Examination Center, The 1st Affiliated Hospital of Anhui Medical University, Hefei, China; ${ }^{2}$ The University Hospital of Wenzhou Medical College, Wenzhou, China; ${ }^{3}$ Division of ECG and Cardiac Function, The 1st Affiliated Hospital of Anhui Medical University, Hefei, China; ${ }^{4}$ School of Environmental Science and Public Health, Wenzhou Medical College, Wenzhou, China and ${ }^{5}$ Department of Clinical Epidemiology, Institute of Industrial Ecological Sciences, University of Occupational and Environmental Health, Kitakyushu City, Japan

6These authors contributed equally to this work.

Correspondence: Professor G Mao, School of Environmental Science and Public Health, Wenzhou Medical College, Chashan University Town, Wenzhou City, 325035, China. E-mail: maogy.net@gmail.com

Received 18 May 2012; revised 26 August 2012; accepted 5 September 2012; published online 17 January 2013 
has recently been widely considered as a more accurate indicator of arteriosclerosis than PWV. ${ }^{6}$ Clinical studies have been conducted during the past few decades to investigate the predictive value of CAVI as an indicator of cardiovascular risk. ${ }^{7}$ Okura et al. ${ }^{8}$ indicated that CAVI is a useful clinical marker for evaluating atherosclerosis and arteriolosclerosis in patients with essential hypertension. Although previous studies have selected CAVI $\geqslant 9.0$ as the diagnostic cutoff point for clinical arteriosclerosis, 9,10 this cutoff point is not widely accepted. The lack of a reliable diagnostic criterion for CAVI hampers proper clinical screening for arteriosclerosis with CAVI and impedes prompt treatment of CVD. The aim of this study was to determine a proper diagnostic cutoff point that would best serve as the diagnostic marker for prediction of arteriosclerosis using CAVI in Chinese patients. Based on data on carotid atherosclerosis early detection and CAVI scores, receiver operating characteristic (ROC) analysis was used to determine an appropriate diagnostic cutoff point.

\section{METHODS}

\section{Study population and procedure}

From May 2010 to September 2011, a total of 328 subjects, 19-90 years of age, participated in an ordinary checkup in the medical examination center of the 1st affiliated hospital of Anhui Medical University. None of the subjects was a patient, and none had obvious signs or symptoms of coronary stenosis or cerebral vascular disease. The participants were recruited from 19 counties of Anhui province, China. After carefully reviewing the protocol, the Ethics Committee of the 1st affiliated hospital of Anhui Medical University approved the study. All of the participants were informed of the aim and procedures in detail and asked to give written informed consent. Of the 328 subjects, 183 (55.79\%) were diagnosed with arteriosclerosis in the carotid arteries and were defined as the carotid arteriosclerosis group. The other 145 (44.21\%) subjects without carotid arteriosclerosis were defined as the non-sclerosis group.

After informed consent was obtained, all subjects were asked to fast overnight for at least $10-12 \mathrm{~h}$. Data collection was conducted by trained investigators who strictly followed the standard operation procedure. Each eligible participant was interviewed using a standardized questionnaire designed specifically for this study to collect information including birth date, CVD history, nutrient intake, cigarette smoking and alcohol drinking habits. Height was measured without shoes on to the nearest $0.1 \mathrm{~cm}$ on a portable stadiometer. Weight was measured to the nearest $0.1 \mathrm{~kg}$ while the subject was wearing light indoor clothing without shoes. Body mass index (BMI) was calculated according to the following equation: $\mathrm{BMI}=$ weight (kilograms)/ height/height (meters).

\section{Measurement of CAVI}

CAVI measurements were performed during $0800-1000 \mathrm{~h}$ in a quiet environment after at least $10 \mathrm{~h}$ of fasting. After resting for at least $15-30 \mathrm{~min}$, CAVI was measured in duplicate with a VS-1000 arteriosclerosis detector (Fukuda Denshi, Tokyo, Japan) by well-trained technicians in the medical examination center. In brief, with the subject supine, the head in midline position, and limbs extend and relaxed, cuffs were applied to bilaterally to the upper arms and ankles. Electrodes were fixed to each wrist, and a heart sound sensor was placed on the second intercostal sternum. In stable baseline cardiechema, the audible 1st and 2nd heart sound would be highly pronounced. The arteriosclerosis detector was equipped with both measurement and calculation systems to calculate the CAVI automatically. CAVI was calculated by the following formula ${ }^{11}$

$$
\mathrm{CAVI}=a\left[(2 \rho / \mathrm{DP}) \times \ln \left(P_{\mathrm{s}} / P_{\mathrm{d}}\right) \mathrm{PWV}^{2}\right]+b
$$

where $P_{\mathrm{s}}$ means systolic $\mathrm{BP}, P_{\mathrm{d}}$ is diastolic $\mathrm{BP}, \mathrm{PWV}$ is the pulse wave velocity, $\Delta P$ is $P_{\mathrm{s}}-P_{\mathrm{d}}, \rho$ is blood density and $a$ and $b$ are constants. The average coefficients of variation of CAVI in the carotid arteriosclerosis and non-sclerosis groups were 5.75 and $3.81 \%$, respectively, indicating that CAVI had good reproducibility and could be widely used for carotid arteriosclerosis screening.

\section{Carotid ultrasound detection}

Carotid ultrasound detection was performed with a XARIO SSA-660A color Doppler ultrasound diagnostic apparatus (Toshiba, Tokyo, Japan) by trained physicians. After at least $5-10 \mathrm{~min}$ rest and with the subject in a supine position, carotid ultrasound detection was conducted in a quiet environment. The intima-media thickness (IMT) and the areas and numbers of arteriosclerotic plaques of the carotid artery were measured. The area of cross intimamedia was also calculated. The diagnostic criteria of arteriosclerosis ${ }^{12}$ were defined as any of the following: (1) roughness and thickening of the intima of the carotid artery and carotid artery IMT $>1 \mathrm{~mm}$; (2) thickness of the carotid artery bifurcation $>1.2 \mathrm{~mm}$; or (3) occurrence of plaque or lumen stenosis. Those whose carotid ultrasound detection results met the diagnostic criteria were characterized into the carotid arteriosclerosis group, and the others were defined as the non-sclerosis group.

\section{Other measurements}

After $10-12 \mathrm{~h}$ of fasting, a venous blood sample was obtained from each participant with tubes containing EDTA (for plasma) or no EDTA (for serum) at baseline. Plasma and serum samples were separated within $15 \mathrm{~min}$ of collection and were analyzed within $30 \mathrm{~min}$ or stored at $-86^{\circ} \mathrm{C}$. Blood samples were collected for the measurement of fasting plasma glucose (FPG); serum lipids, including total cholesterol, high-density lipoprotein and triglycerides; creatinine; serum urea nitrogen; Apoprotein-A and Apoprotein-B. All of these tests were performed in duplicate by well-trained technicians in the central laboratory of the 1st hospital of Anhui Medical University, using standard reagents and an automatic biochemistry analyzer. Low-density lipoprotein cholesterol was calculated by Friedewald's equation. All of the inter-assay coefficients of variation were $<5.0 \%$.

\section{Statistical analysis}

Double data entry and data cleaning, including verification of the completeness and plausibility of the source data, were performed using Epidata (version 3.1, Odense, Denmark). The normality of continuous data distribution was assessed with the Kolmogorov-Smirnov test before statistical analysis. If the data had a normal or near normal distribution, then an independent sample $t$ test was performed to compare the characteristics of the subjects between the two groups. Otherwise, the Mann-Whitney $U$-test was used. A $\chi^{2}$ test (or Fisher's exact test) for categorical variables was applied to compare the equalization and comparability of participants in the two groups.

The association between CAVI and carotid arteriosclerosis was examined by Spearman's correlation analyses, $\chi^{2}$ test and generalized linear models. Then, we stratified the 328 subjects as an average into three subgroups according to their CAVI scores and assigned them to two subgroups using the cutoff point based on the ROC analyses. With adjustments for age, gender, BMI, lowdensity lipoprotein cholesterol, FPG, BP, creatinine, education, occupation, psychological tension, cigarette smoking and alcohol drinking, generalized linear models were applied to determine whether the association between carotid arteriosclerosis and a CAVI increase was true or not. As the CAVI cutoff point for predicting arteriosclerosis, we chose the point on the ROC curve that represented the largest sum of sensitivity and specificity. The ability to identify arteriosclerosis correctly by CAVI was measured by the area under the ROC curve (AUC), sensitivity and specificity. We also performed an analysis of the joint associations of age and CAVI for the risk of carotid arteriosclerosis. All tests were two-sided and $P \leqslant 0.05$ was set as statistically significant. Data management and all statistical analyses were performed using $\mathrm{R}$ version 2.14.0 (Copyright 2011 the R Foundation for Statistical Computing) and Empower(R) Statistical Data Analyzer for Windows (Copyright 2009 X\&Y Solutions, Boston, MA, USA).

\section{RESULTS}

\section{Characteristics of participants}

A total of 328 subjects were recruited from the medical examination center of the 1st hospital of Anhui Medical University during May 2010 to September 2011. The demographic and clinical characteristics of the subjects with and without carotid arteriosclerosis are shown in 
Table 1 Epidemiological and clinical characteristics of subjects ${ }^{\mathrm{a}}$

\begin{tabular}{|c|c|c|c|}
\hline Variables & Non-sclerosis & Carotid arteriosclerosis & $P$ \\
\hline Age, years & $36.00(31.00-45.00)$ & $63.00(56.00-69.00)$ & $<0.001$ \\
\hline $\mathrm{SBP}, \mathrm{mm} \mathrm{Hg}$ & $116.00(104.50-126.00)$ & $130.00(120.00-143.50)$ & $<0.001$ \\
\hline DBP, $\mathrm{mm} \mathrm{Hg}$ & $76.00(70.00-84.00)$ & 80.00 (76.00-90.00) & $<0.001$ \\
\hline CAVI & $7.15(6.65-7.70)$ & $8.80(7.95-9.85)$ & $<0.001$ \\
\hline HDL-C, mmoll-1 & $1.44(1.26-1.70)$ & $1.46(1.20-1.70)$ & 0.971 \\
\hline LDL-C, mmoll-1 & $2.57(2.01-2.95)$ & $2.81(2.38-3.39)$ & $<0.001$ \\
\hline Apoprotein-A, mmoll-1 & $1.37(1.16-1.56)$ & $1.32(1.13-1.57)$ & 0.454 \\
\hline Apoprotein-B, mmoll-1 & $0.82 \pm 0.16$ & $0.88 \pm 0.17$ & 0.003 \\
\hline $\mathrm{FPG}, \mathrm{mmoll}^{-1}$ & $5.19(4.87-5.60)$ & $5.66(5.22-6.19)$ & $<0.001$ \\
\hline Urea nitrogen, $\mu \mathrm{mol} \mathrm{I}^{-1}$ & $5.08(4.47-6.00)$ & $5.63(4.86-6.86)$ & $<0.001$ \\
\hline Female & $77(53.1)$ & $50(27.3)$ & \\
\hline Education & & & 0.019 \\
\hline Junior high school or below & $45(31.0)$ & $80(43.7)$ & \\
\hline High school or above & $100(69.0)$ & $103(56.3)$ & \\
\hline Occupation & & & 0.002 \\
\hline Manual worker & $100(69.0)$ & $96(52.5)$ & \\
\hline Non-manual worker & $45(31.0)$ & $87(47.5)$ & \\
\hline Work intensity & & & 0.001 \\
\hline Low & $72(49.7)$ & $123(67.2)$ & \\
\hline High & $73(50.3)$ & $60(32.8)$ & \\
\hline Psychological tension & & & $<0.001$ \\
\hline No & $41(28.5)$ & $113(61.7)$ & \\
\hline Passive smoking & & & 0.010 \\
\hline No & $35(24.6)$ & $68(38.2)$ & \\
\hline often & $107(75.4)$ & $110(61.8)$ & \\
\hline Alcohol drinking ${ }^{c}$ & & & 0.075 \\
\hline No & $70(48.3)$ & $70(38.5)$ & \\
\hline Yes & 75 (51.7) & $112(61.5)$ & \\
\hline Menopause & & & $<0.001$ \\
\hline No & $71(92.2)$ & $11(22.4)$ & \\
\hline Yes & $6(7.8)$ & $38(77.6)$ & \\
\hline
\end{tabular}

Abbreviations: BMI, body mass index; CAVI, cardio-ankle vascular index; DBP, diastolic blood pressure; FPG, fasting plasma glucose; HDL-C, high-density lipoprotein cholesterol; LDL-C, low-density lipoprotein cholesterol; TC, total cholesterol; TG, triglyceride; SBP, systolic blood pressure.

${ }^{a}$ Continuous data meeting normal distribution were described as mean \pm s.d. and an independent $t$ test was performed to compare the differences between the two groups, otherwise median (Q1-Q3) and the Mann-Whitney U-test were used. Categorical data were described as number of cases (\%) and a $\chi^{2}$ test or Fisher's exact test was selected to compare the differences. ${ }^{b}$ Cigarette smoking was defined as one time or current smoker.

CAlcohol drinking was defined as more than one time per week or current drinker.

Table 1 . Of the 328 subjects (38.72\% women, $19-90$ years of age), $98.48 \%$ were of Han nationality, and $55.79 \%$ had carotid arteriosclerosis but none with apparent lumen stenosis. The mean BMI was $23.60 \pm 3.28 \mathrm{~kg} \mathrm{~m}^{-2}$. As the CAVI score distribution was positively skewed, the Mann-Whitney $U$-test was applied to compare the difference between subjects with or without carotid arteriosclerosis. The median (Q1-Q3) of CAVI in the two groups was 8.80 (7.95-9.85) and 7.15 (6.65-7.70), respectively. The CAVI score for carotid arteriosclerosis participants was significantly higher than that for non-sclerosis subjects $(P<0.001)$. This finding indicates that CAVI significantly associated with the degree of carotid arteriosclerosis. Table 1 also shows that participants in each group are not well balanced $(P<0.05)$, which indicates that age, gender, BMI, BP, dyslipidemia, hyperglycemia, renal dysfunction, psychological tension, 
cigarette smoking and alcohol drinking, may significantly associated with carotid arteriosclerosis.

\section{Association between carotid arteriosclerosis and CAVI}

To assess the association between CAVI and arteriosclerosis, the correlation between CAVI and the IMT was examined by Spearman correlation analysis because of the skewed distributions. In the stratified analyses, a significant positive correlation between IMT and CAVI was observed in participants with carotid arteriosclerosis $(r=0.361, P<0.001)$, whereas this relationship was not present for those without carotid arteriosclerosis $(r=-0.006, \quad P=0.946)$ (Figure 1). Furthermore, we also explored the association between CAVI and other carotid artery parameters, such as rough and thickening intima, thickening intima of bifurcation and the number of plaques. In participants with $\mathrm{CAVI}<8.0$ and $\mathrm{CAVI} \geqslant 8.0$, the prevalence of thickening of the intima was 60.82 and $80.25 \%$, respectively, and the prevalence of carotid arterial plaques was 4.09 and $15.29 \%$, respectively. Participants with higher CAVI scores had higher odds ratios (ORs) for the presence of thickening of the intima $(\mathrm{OR}, 2.62 ; 95 \%$ confidence interval (CI), 1.59-4.31) and carotid artery plaques (OR, 4.23; 95\% CI, 1.77-10.12), compared with those with CAVI $<8.0$. Our data clearly showed that CAVI is significantly associated with the risk of carotid arteriosclerosis. The higher the CAVI level, the higher the number of carotid artery plaques and the greater the risk of carotid arteriosclerosis (Table 2). Figure 2 also clearly shows that the risk of carotid arteriosclerosis correlates with the CAVI score in the male, female and pooled populations. As the CAVI score increases, the probability of carotid arteriosclerosis also increases greatly.

It is clear that CAVI is not the only factor that is an indicator of arteriosclerosis probability; many other factors are also significantly associated with risk of arteriosclerosis. Because of these potential confounding biases, it is considered valuable to explore the association between CAVI and carotid arteriosclerosis after adjusting for these potential confounding factors. We first divided the 328 subjects into three strata according to CAVI values: 5.15-7.40 (Low), 7.45-8.65 (Medium) and 8.66-13.60 (High). The prevalence of carotid arteriosclerosis among the 'Low', 'Medium' and 'High' groups is 19.8, 57.0 and $90.9 \%$, respectively (Table 4). These data show that the higher the CAVI score, the greater the risk of carotid arteriosclerosis. Compared with the low group, the ORs $(95 \% \mathrm{CI})$ of the medium and high groups are $5.4(2.9,9.8)$ and $40.5(18.2,90.1)$, respectively. After adjustments for some potential risk factors, the ORs ( $95 \% \mathrm{CI})$ of the two groups were $2.1(1.0,4.7)$ and $5.2(1.9,14.0)$, respectively, in model I, and $2.2(1.0,4.9)$ and $4.4(1.5,13.3)$, respectively, in model II, confirming that CAVI is an independent risk factor for carotid arteriosclerosis and can be defined as a marker for prediction of arteriosclerosis (trend test $P<0.001$ ) (Table 4).

To explore an optimal CAVI cutoff point for arteriosclerosis, we chose the point that, based on ROC analyses, represents the largest sum of sensitivity and specificity. The AUC (95\% CI), sensitivity and specificity for CAVI in predicting arteriosclerosis are presented in Figure 3 and Table 3. The AUC (95\% CI) for CAVI is $0.79(0.72,0.86)$ for males, $0.90(0.85,0.94)$ for females and $0.86(0.82,0.90)$ for all 328 subjects. Figure 2 and Table 5 also show that the probability of carotid arteriosclerosis is significantly and positively associated with age. The AUC $(95 \%$ CI $)$ for age is $0.87(0.81,0.93)$ for males, $0.97(0.93,1.00)$ for females and $0.92(0.89,0.95)$ for the pooled population. The optimal cutoff points according to the ROC curve are as follows: 8.18 (males), 7.88 (females) and 8.03 (pooled) for CAVI; and 52.92 (males), 44.64 (females) and 46.23(pooled) for age (Table 3). Based on the result of the ROC analyses, we chose CAVI $\geqslant 8.0$ and age $>46$ as the cutoff points. After adjustments for some potential risk factors, compared with CAVI $<8.0$ or age $\leqslant 46$ group, the OR $(95 \% \mathrm{CI})$ is 3.3 $(1.6,6.9)$ in model I and $2.8(1.3,6.2)$ in model II for CAVI (Table 4$)$,

Table 2 The risk of carotid arteriosclerosis findings and CAVI level ${ }^{\mathrm{a}}$

\begin{tabular}{|c|c|c|c|c|}
\hline Variables & $\begin{array}{l}C A V I<8.0 \\
(N=171)\end{array}$ & $\begin{array}{l}C A V I \geqslant 8.0 \\
(N=157)\end{array}$ & OR $(95 \% \mathrm{Cl})$ & $P$ \\
\hline \multicolumn{5}{|c|}{ Thickening of intima of bifurcation } \\
\hline No & $67(39.18)$ & $31(19.75)$ & Ref & - \\
\hline Yes & $104(60.82)$ & $126(80.25)$ & $2.62(1.59,4.31)$ & $<0.001$ \\
\hline \multicolumn{5}{|c|}{ Carotid artery plaque } \\
\hline No & $164(95.91)$ & $133(84.71)$ & Ref & - \\
\hline Yes & $7(4.09)$ & $24(15.29)$ & $4.23(1.77,10.12)$ & 0.001 \\
\hline \multicolumn{5}{|c|}{ Numbers of plaques } \\
\hline 0 & 164 (95.91) & $133(84.71)$ & Ref & - \\
\hline 1 & $5(2.92)$ & $15(9.55)$ & $3.70(1.31,10.44)$ & 0.013 \\
\hline $2-3$ & $2(1.17)$ & $9(5.74)$ & $5.55(1.18,26.12)$ & 0.030 \\
\hline \multicolumn{5}{|c|}{ Lumen stenosis } \\
\hline No & $171(100.00)$ & $157(100.00)$ & - & - \\
\hline Yes & $0(0.00)$ & $0(0.00)$ & - & - \\
\hline
\end{tabular}

Abbreviations: $\mathrm{Cl}$, confidence interval; OR, odds ratio.

a CAVI indicates cardio-ankle vascular index; $N$ indicates sample size of each group.
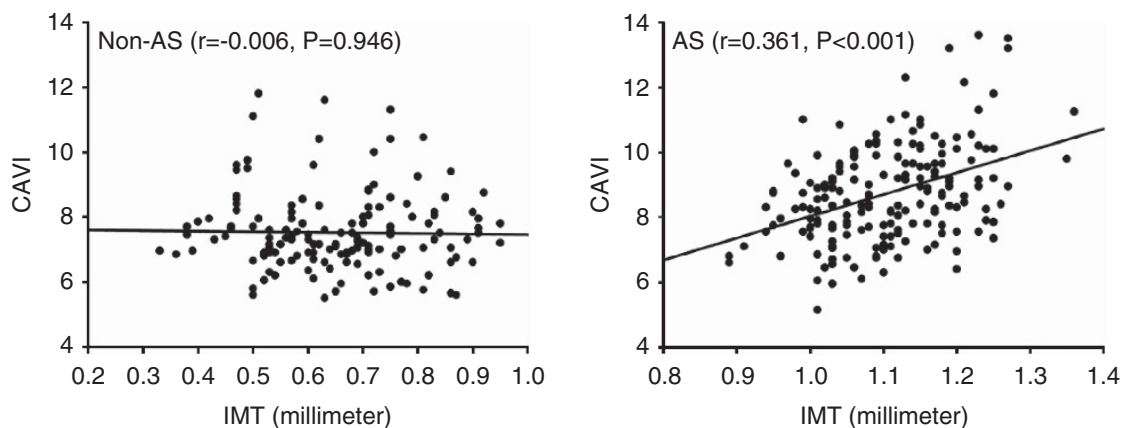

Figure 1 The dot-plot graph between CAVI with IMT. CAVI, cardio-ankle vascular index; IMT, intima-media thickness; Non-AS, non-arteriosclerotic group (population without carotid arteriosclerosis); AS, carotid arteriosclerosis group (population with carotid arteriosclerosis). R, spearman correlation coefficients. 


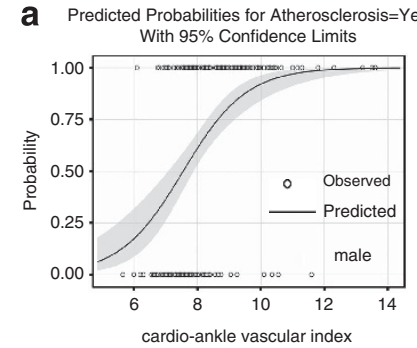

d Predicted Probabilities for Atherosclerosis=Yes

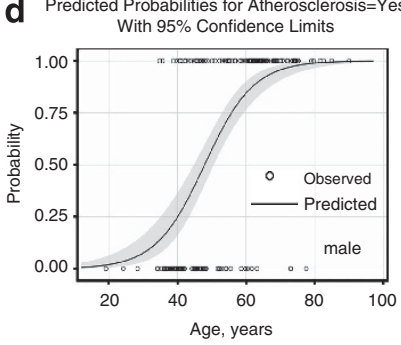

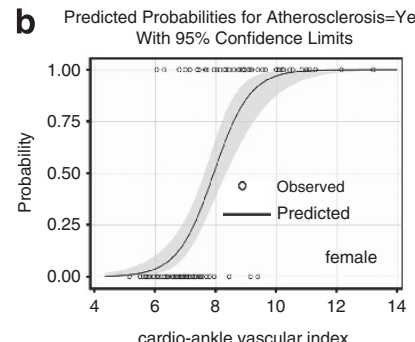

e Predicted Probabilities for Atherosclerosis=Yes

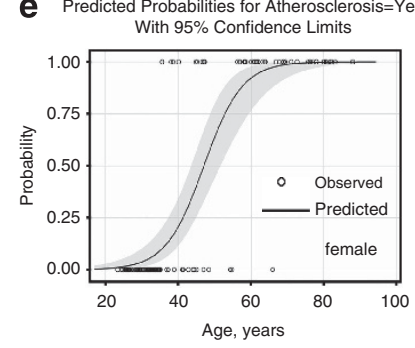

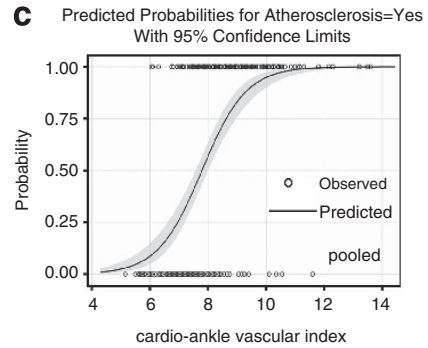

f Predicted Probabilities for Atherosclerosis=Yes

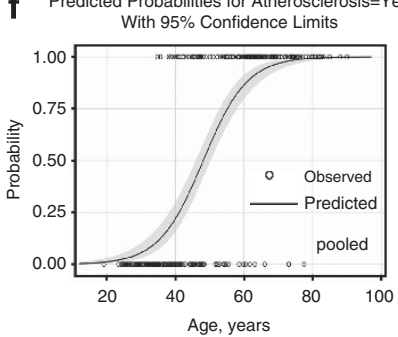

Figure 2 Risk of carotid arteriosclerosis for CAVI and age. a, b and $\mathbf{c}$ Predicted probability for arteriosclerosis by CAVI in male, female and pooled group. $\mathbf{d}, \mathbf{e}$ and $\mathbf{f}$ Predicted probability for arteriosclerosis by age in male, female and pooled group.
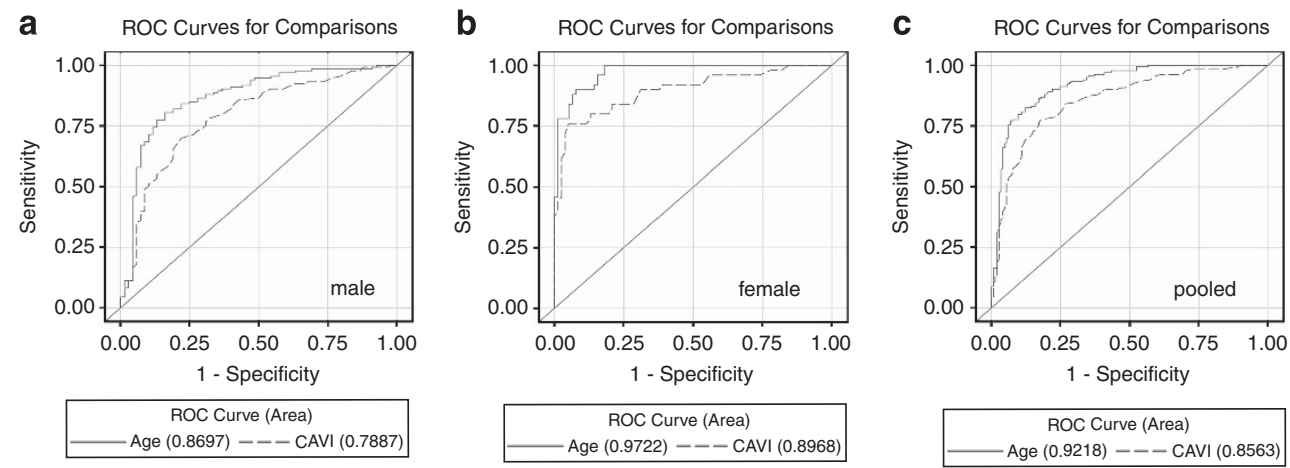

Figure 3 Receiver operating characteristic (ROC) curve and area under the ROC curve (AUC) showing the ability of cardio-ankle vascular index (CAVI) or age to predict carotid arteriosclerosis. Panels $\mathbf{a}, \mathbf{b}$ and $\mathbf{c}$ indicate the ability of carotid arteriosclerosis predition with age and CAVI in male, female and pooled group.

and $11.7(5.6,24.6)$ in model I and $10.8(4.9,23.9)$ in model II for age (Table 5). CAVI and age seem to be good markers for carotid arteriosclerosis screening. Furthermore, for carotid arteriosclerosis, the AUC for age is significantly larger than that for CAVI $(P<0.05$, Table 3).

Joint association of age and CAVI for prediction of arteriosclerosis Because CAVI and age can both be used for atherosclerosis prediction, it is necessary to test for interaction between CAVI and age. Table 6 shows the joint association of CAVI and age with the risk of carotid arteriosclerosis. The prevalences for the four groups are as follows: $12.8 \%$ for subjects with age $\leqslant 46.0$ and CAVI $<8.0$ (Reference), $46.2 \%$ for those with age $\leqslant 46.0$ but CAVI $\geqslant 8.0$ (Group 1 ), $71.7 \%$ for subjects with age $>46$ but CAVI $<8.0$ (Group 2) and $88.9 \%$ for both age $>46.0$ and $\mathrm{CAVI} \geqslant 8.0$ (Group 3). After adjustment for the major covariates, the risk of carotid arteriosclerosis increases greatly in the other three groups compared with the reference group. In model I, the OR $(95 \% \mathrm{CI})$ is $3.1(0.8,12.0)$ for group $1,12.1(4.9,29.8)$ for group 2 and $41.0(17.6,95.5)$ for group 3 . A similar pattern can be seen in model II. A significant correlation was found between age and
CAVI. Notably, the highest risk was found in subjects with both age $>46$ and CAVI $\geqslant 8.0$. CAVI and age appear to act synergistically to increase the risk of carotid arteriosclerosis.

\section{DISCUSSION}

Atherosclerosis has emerged as a major risk factor for CVD, which is the leading health problem in both developed and developing countries, such as China. ${ }^{13}$ It is of great clinical and public health importance to develop a cutoff value for diagnostic screening for carotid arteriosclerosis, which is beneficial for early arteriosclerosis detection and treatment. Although previous studies have shown that the CAVI score is independently and positively associated with the risk of arteriosclerosis, no widely accepted diagnostic criterion on carotid arteriosclerosis using CAVI has been suggested greatly hampering proper clinical screening of carotid arteriosclerosis with CAVI and impeding prompt, early detection and treatment of CVD. Our study suggests that CAVI $\geqslant 8.0$ may be an optimal criterion for prediction of arteriosclerosis. We also found that both CAVI and age are independently and synergistically associated with the risk of carotid arteriosclerosis. The present study confirms that an increased 
Table 3 Risk of carotid arteriosclerosis by CAVI status

\begin{tabular}{|c|c|c|c|c|c|c|c|c|}
\hline \multirow[b]{2}{*}{ CAVI } & \multirow[b]{2}{*}{$N$} & \multirow[b]{2}{*}{ Cases (\%) } & \multicolumn{2}{|c|}{ Crude } & \multicolumn{2}{|c|}{ Model I } & \multicolumn{2}{|c|}{ Model II } \\
\hline & & & OR $(95 \% \mathrm{Cl})$ & $P$ & OR $(95 \% \mathrm{Cl})$ & $P$ & OR $(95 \% \mathrm{Cl})$ & $P$ \\
\hline \multicolumn{9}{|l|}{ Stratum 1} \\
\hline $5.15 \sim$ & 111 & $22(19.8)$ & $1.0(1.0,1.0)$ & Ref & $1.0(1.0,1.0)$ & Ref & $1.0(1.0,1.0)$ & Ref \\
\hline $7.41 \sim$ & 107 & $61(57.0)$ & $5.4(2.9,9.8)$ & $<0.001$ & $2.1(1.0,4.7)$ & 0.062 & $2.2(1.0,4.9)$ & 0.065 \\
\hline $8.66-13.60$ & 110 & 100 (90.9) & $40.5(18.2,90.1)$ & $<0.001$ & $5.2(1.9,14.0)$ & 0.001 & $4.4(1.5,13.3)$ & 0.009 \\
\hline Trend test & & & & $<0.001$ & & 0.001 & & 0.007 \\
\hline \multicolumn{9}{|l|}{ Stratum 2} \\
\hline$<8.0$ & 171 & $49(28.7)$ & $1.0(1.0,1.0)$ & Ref & $1.0(1.0,1.0)$ & Ref & $1.0(1.0,1.0)$ & Ref \\
\hline$\geqslant 8.0$ & 157 & $134(85.4)$ & $14.5(8.3,25.2)$ & $<0.001$ & $3.3(1.6,6.9)$ & 0.002 & $2.8(1.3,6.2)$ & 0.010 \\
\hline
\end{tabular}

Abbreviations: CAVI, cardio-ankle vascular index; $\mathrm{Cl}$, confidence interval; OR, odds ratio.

$N$ : numbers of subjects in each stratum. Cases (\%): numbers of arterial stiffness and percentage.

Model I: adjusted for age, gender, low-density lipoprotein cholesterol, fasting plasma glucose, blood pressure.

Model II: adjusted for age, gender, body mass index, low-density lipoprotein cholesterol, fasting plasma glucose, blood pressure, creatinine, education, occupation, psychological tension, cigarette smoking and alcohol drinking.

Table 4 Prediction of carotid arteriosclerosis with cutoff points for CAVI and age

\begin{tabular}{|c|c|c|c|c|c|c|c|c|c|}
\hline & $\operatorname{A\cup C}(95 \% \mathrm{Cl})$ & S.e. & Cutoff & Sensitivity & Specificity & $\mathrm{P}^{\mathrm{a}}$ & $\beta$ & S.e. & $\mathrm{Pb}^{\mathrm{b}}$ \\
\hline \multicolumn{10}{|l|}{ Male } \\
\hline Age & $0.87(0.81,0.93)$ & 0.03 & 52.92 & 0.81 & 0.84 & $<0.001$ & - & - & Ref \\
\hline CAVI & $0.79(0.72,0.86)$ & 0.03 & 8.18 & 0.70 & 0.78 & $<0.001$ & -0.08 & 0.03 & 0.001 \\
\hline \multicolumn{10}{|l|}{ Female } \\
\hline Age & $0.97(0.93,1.00)$ & 0.02 & 44.64 & 0.90 & 0.92 & $<0.001$ & - & - & Ref \\
\hline CAVI & $0.90(0.85,0.94)$ & 0.02 & 7.88 & 0.76 & 0.95 & $<0.001$ & -0.08 & 0.03 & 0.018 \\
\hline \multicolumn{10}{|l|}{ Pooled } \\
\hline Age & $0.92(0.89,0.95)$ & 0.02 & 46.23 & 0.88 & 0.80 & $<0.001$ & - & - & Ref \\
\hline CAVI & $0.86(0.82,0.90)$ & 0.02 & 8.03 & 0.73 & 0.85 & $<0.001$ & -0.07 & 0.02 & $<0.001$ \\
\hline
\end{tabular}

Abbreviations: AUC, area under the receiver operating characteristic curve; CAVI, cardio-ankle vascular index; $95 \% \mathrm{Cl}$, 95\% confidence interval.

a $P$-value compared with Null hypothesis: true area $=0.5 ; \beta$ : regression coefficient.

b $P$-value of the area under curve of CAVI compared with age.

Table 5 Risk of carotid ateriosclerosis by age

\begin{tabular}{|c|c|c|c|c|c|c|c|c|}
\hline Age & $N$ & Cases (\%) & OR $(95 \% \mathrm{Cl})$ & $P$ & OR $(95 \% \mathrm{Cl})$ & $P$ & OR $(95 \% \mathrm{Cl})$ & $P$ \\
\hline $19 \sim$ & 114 & $12(10.5)$ & $1.0(1.0,1.0)$ & Ref & $1.0(1.0,1.0)$ & Ref & $1.0(1.0,1.0)$ & Ref \\
\hline $42 \sim$ & 102 & $65(63.7)$ & $14.9(7.3,30.7)$ & $<0.001$ & $8.4(3.7,19.0)$ & $<0.001$ & $8.1(3.5,18.8)$ & $<0.001$ \\
\hline $62-90$ & 112 & $106(94.6)$ & $150.2(54.3,415.2)$ & $<0.001$ & $54.8(16.8,179.0)$ & $<0.001$ & $52.3(14.9,183.5)$ & $<0.001$ \\
\hline \multicolumn{9}{|l|}{ Stratum 2} \\
\hline$\leqslant 46$ & 138 & 22 (15.9) & $1.0(1.0,1.0)$ & Ref & $1.0(1.0,1.0)$ & Ref & $1.0(1.0,1.0)$ & Ref \\
\hline$>46$ & 190 & $161(84.7)$ & $29.3(16.0,53.5)$ & $<0.001$ & $11.7(5.6,24.6)$ & $<0.001$ & $10.8(4.9,23.9)$ & $<0.001$ \\
\hline
\end{tabular}

Abbreviations: CAVI, cardio-ankle vascular index; 95\% CI, 95\% confidence interval; OR, odds ratio.

$N$ : numbers of subjects in each stratum. Cases (\%): numbers of arterial stiffness and percentage.

Model I: adjusted for CAVI, gender, low-density lipoprotein cholesterol, fasting plasma glucose, and blood pressure.

Model II: adjusted for CAVI, gender, body mass index, low-density lipoprotein cholesterol, fasting plasma glucose, blood pressure, creatinine, education, occupation, psychological tension, cigarette smoking and alcohol drinking.

CAVI score is indicative of a significantly increased risk of arteriosclerosis. CAVI can be used as a good marker to identify individuals with an extensive arteriosclerosis burden who are at a high risk for CVD and who may therefore benefit from more aggressive interventions. Subjects with $\mathrm{CAVI} \geqslant 8.0$ and age $>46$ years have the highest risk of carotid arteriosclerosis, whereas those with $\mathrm{CAVI}<8.0$ 
Table 6 Interaction of age and CAVI for risk of carotid arteriosclerosis

\begin{tabular}{|c|c|c|c|c|c|c|c|c|c|}
\hline \multirow[b]{2}{*}{ Age } & \multirow[b]{2}{*}{ CAVI } & \multirow[b]{2}{*}{$N$} & \multirow[b]{2}{*}{ Cases (\%) } & \multicolumn{2}{|l|}{ Crude } & \multicolumn{2}{|l|}{ Model I } & \multicolumn{2}{|c|}{ Model II } \\
\hline & & & & OR $(95 \% \mathrm{Cl})$ & $P$ & OR $(95 \% \mathrm{Cl})$ & $P$ & OR $(95 \% \mathrm{Cl})$ & $P$ \\
\hline$\leqslant 46$ & $<8$ & 125 & $16(12.8)$ & $1.0(1.0,1.0)$ & Ref & $1.0(1.0,1.0)$ & Ref & $1.0(1.0,1.0)$ & Ref \\
\hline$\leqslant 46$ & $\geqslant 8$ & 13 & $6(46.2)$ & $5.8(1.7,19.6)$ & 0.004 & $3.1(0.8,12.0)$ & 0.106 & $3.0(0.7,12.2)$ & 0.123 \\
\hline$>46$ & $<8$ & 46 & $33(71.7)$ & $17.3(7.5,39.6)$ & $<0.001$ & $12.1(4.9,29.8)$ & $<0.001$ & $12.4(4.8,31.8)$ & $<0.001$ \\
\hline$>46$ & $\geqslant 8$ & 144 & $128(88.9)$ & $54.5(26.0,114.1)$ & $<0.001$ & $41.0(17.6,95.5)$ & $<0.001$ & $36.3(14.4,91.7)$ & $<0.001$ \\
\hline Trend test & & & & & $<0.001$ & & $<0.001$ & & $<0.001$ \\
\hline
\end{tabular}

Abbreviations: CAVI, cardio-ankle vascular index; 95\% CI, 95\% confidence interval; OR, odds ratio.

$N$ : numbers of subjects in each stratum. Cases (\%): numbers with arterial stiffness and percentage.

Model I: adjusted for gender, low-density lipoprotein cholesterol, fasting plasma glucose and blood pressure.

Model II: adjusted for gender, body mass index, low-density lipoprotein cholesterol, fasting plasma glucose, blood pressure, creatinine, education, occupation, psychological tension, cigarette

smoking and alcohol drinking.

and age $\leqslant 46$ years have the lowest risk. The joint association of CAVI and age with carotid arteriosclerosis appears to be synergistic. Our findings seem to be more robust after adjustment for some important covariates.

Carotid arteriosclerosis is the principal pathophysiologic change known to strongly and independently contribute to $\mathrm{CVD}^{4,14}$ and can be measured visually (by carotid/vertebral artery solography, cerebral angiography and magnetic resonance angiography $)^{15}$ and functionally (by PWV, stiffness parameter $\beta$ and CAVI). ${ }^{6,16,17}$ In general, functional measurements are widely accepted, mainly because of their non-invasiveness, inexpensiveness and ease of use. Among the measurements of carotid arteriosclerosis, one early method to quantitatively estimate the extent of arteriosclerosis is $\mathrm{PWV},{ }^{18}$ which was first introduced by Bramwell and Hill ${ }^{18}$ and developed as early as the 1920s. Despite being widely used as a means of carotid arteriosclerosis assessment, PWV is significantly affected by many factors, such as age, BP, hyperglycemia, dyslipidemia, cigarette smoking and alcohol consumption, which makes clinical interpretation difficult. Hayashi et al. ${ }^{19}$ first introduced the stiffness parameter $\beta$, which is independent of BP, in the 1980s, and it has since been developed as a marker for stiffness evaluation. ${ }^{20}$ However, the major shortcoming of the stiffness parameter $\beta$ is that it requires measurement of an inner arterial diameter at systole and diastole by ultrasound echography, which greatly lessens its clinical availability. To overcome the limitations of PWV and the stiffness parameter $\beta$, CAVI was introduced clinically by Shirai et al. ${ }^{6}$ as a novel, simple, non-invasive measurement in the assessment of arteriosclerosis, which quantitatively reflects arteriosclerosis of the aorta, femoral and tibial arteries based on Bramwell-Hill's equation and stiffness parameter. ${ }^{21}$ CAVI is easy to perform; only requires measurement of BP and pulse wave data, independent of BP; and has adequate reproducibility for wide clinical use. Previous studies have found that CAVI was strongly associated with the presence of severe coronary arteriosclerosis ${ }^{22}$ and could be used as a screening tool for arteriosclerosis. ${ }^{23}$ As shown in Table 4 of our study, after adjustment for some major confounding factors, a linear association exists between CAVI scores and carotid arteriosclerosis in both male and female participants. Our data confirm that CAVI is an independent predictor of carotid arteriosclerosis, which is consistent with the findings of western studies. ${ }^{24,25}$ Our study also confirms that CAVI scores reflect the extent of carotid arteriosclerosis and can be used for the early detection of arteriosclerosis as described in previous studies. ${ }^{26,27}$

As a widely accepted optimal method to determine a cutoff point, ROC analyses represent the largest sum of sensitivity and specificity and can best serve as the diagnostic marker. Thus, we performed ROC analyses to find the optimal criterion for predicting carotid arteriosclerosis with CAVI in Chinese populations. Based on the ROC curve, we found that carotid arteriosclerosis is strongly and positively associated with CAVI in males, females and the overall population. After adjustment for major potential risk factors, CAVI $\geqslant 8.0$ was found to be an optimal cutoff point for predicting carotid arteriosclerosis with CAVI in the Chinese population. Furthermore, the present study also found that age is significantly and independently associated with arteriosclerosis and can be used as another indicator in arteriosclerosis screening and early detection. Based on the results of ROC analysis, the cutoff point for age appears to be age $>46$ years old. Compared with younger participants, the population older than 46 years has an increased risk of arteriosclerosis of at least 980\% (OR, 10.8; 95\% CI, 4.9-23.9, $P<0.001$ ). Individuals younger than that age are considered to have an average risk for atherosclerosis. With the great improvement in living standards over the past few decades, there is an increasing risk of CVD in younger people as a result of cigarette smoking, the alarming increase in obesity, sedentary lifestyle, lack of physical activity and greatly changed dietary habits.

As both CAVI and age are significantly and positively associated with the extent of arteriosclerosis and risk of CVD, and both can be good markers for carotid arteriosclerosis screening, it is necessary to determine whether there is an interaction between CAVI and age in prediction of carotid arteriosclerosis. Unfortunately, there have been no reports of whether CAVI and age are correlated for prediction of carotid arteriosclerosis. To the best of our knowledge, this is the first study to assess the joint association of CAVI and age for predicting arteriosclerosis. The data from the present study show a significantly synergistic effect between CAVI and age in the predicting arteriosclerosis. As CAVI score and age increase, the risk of carotid arteriosclerosis increases strongly (trend test $P<0.001$ ). The highest risk is found in subjects with both age $>46$ and higher CAVI scores $(\mathrm{CAVI} \geqslant 8.0)$. CAVI and age appear to act synergistically to increase the risk of carotid arteriosclerosis. Furthermore, our data also suggest that, to some extent, age may be a more superior predictor of subclinical aortic arteriosclerosis than CAVI.

Potential limitations of our study should also be noted. First, this was a hospital-based cross-sectional study, and the subjects came from only one hospital. Selection bias may be inevitable. However, the 328 participants, recruited from an ordinary checkup in the medical examination center of the 1st affiliated hospital of Anhui Medical University, came from 19 counties in Anhui province and therefore the selection bias could not be quite large. Second, all subjects in our study were recruited from an ordinary checkup. None was a patient, and none had obvious signs or symptoms of coronary stenosis or 
cerebral vascular disease. We used only arteriosclerotic findings in the carotid artery to define whether someone had arteriosclerosis. This may have led to some bias. Carotid arteriosclerosis can generally be defined as arteriosclerosis findings in the carotid artery or findings related to coronary stenosis, renal function or cerebral vascular disease. In all of these findings, carotid atherosclerosis can easily be found in its early stage, which will not have any apparent signs and symptoms of coronary stenosis or cerebral vascular diseases. Currently, carotid ultrasound detection is widely used in the early detection of arteriosclerosis, which is important for the prevention and control of arteriosclerosis and CVD. Third, although the study was designed to recruit participants from a wide area, the study participants were volunteers $(98.48 \%$ are Han nationality) and may not be truly representative of the general population. The results of this cross-sectional study may not be applicable to other racial or ethnic subgroups. Additional large studies, especially prospective cohort studies that include a more representative selection of participants, are warranted to determine whether CAVI $\geqslant 8.0$ is an optimal criterion for screening subjects with a high risk of CVD. Owing to the relatively large sample size of our study, the use of ROC analyses to determine the diagnostic cutoff point and the adjustment for some important covariates using the generalized linear model, our findings seem to be plausible despite these limitations. Indeed, even though our study is limited in several respects, it raises many questions for future studies.

In conclusion, the present study suggests that the optimal cutoff point for predicting carotid arteriosclerosis using CAVI should be $\mathrm{CAVI} \geqslant 8.0$. Our results confirm that both CAVI score and age are independent predictors of the risk of carotid arteriosclerosis and suggest that age may be a more superior predictor of the extent of subclinical aortic arteriosclerosis than CAVI. Furthermore, our study also demonstrates a significant synergistic effect between age and CAVI for predicting arteriosclerosis. CAVI and age appear to synergistically predict the risk of carotid arteriosclerosis. Older subjects with higher CAVI scores have a higher risk of CVD.

\section{CONFLICT OF INTEREST}

The authors declare no conflict of interest.

\section{ACKNOWLEDGEMENTS}

We are grateful to all study participants and their relatives for their contributions. This study was supported by grants from the Anhui Provincial Natural Science Research Project of University (No. KJ2010B279) and Zhejiang Provincial Natural Science Funding (No. Y2110454). We also want to thank Professor Niall Galbraith for his careful and thorough revision of the manuscript.

1 World Health Organization. The World Health Report 2011. http://www.who.int/gho/ publications/world_health_statistics/EN_WHS2011_Full.pdf. Accessed 2012.

2 World Health Organization. The Global Burden of Disease (2010) Study. http://www.who.int/healthinfo/global_burden_disease/GBD_2005_study/en/index.html. Accessed 2012.

3 Nurnberger J, Kribben A, Philipp T, Erbel R. [Arterial compliance (stiffness) as a marker of subclinical atherosclerosis]. Herz 2007; 32: 379-386.
4 Boos CJ, Lane DA, Karpha M, Beevers DG, Haynes R, Lip GY. Circulating endothelial cells, arterial stiffness, and cardiovascular risk stratification in hypertension. Chest 2007: 132: 1540-1547.

5 Namekata T, Moore D, Suzuki K, Mori M, Hatano S, Hayashi C, Abe N, Hasegawa M. [A study of the association between the aortic pulse wave velocity and atherosclerotic risk factors among Japanese Americans in Seattle, USA]. Nihon Koshu Eisei Zasshi 1997; 44: 942-951.

6 Shirai K, Utino J, Otsuka K, Takata M. A novel blood pressure-independent arterial wall stiffness parameter: cardio-ankle vascular index (CAVI). J Atheroscler Thromb 2006; 13: 101-107.

7 Shirai K, Hiruta N, Song M, Kurosu T, Suzuki J, Tomaru T, Miyashita Y, Saiki A, Takahashi M, Suzuki K, Takata M. Cardio-ankle vascular index (CAVI) as a novel indicator of arterial stiffness: theory, evidence and perspectives. J Atheroscler Thromb 2011; 18: 924-938.

8 Okura T, Watanabe S, Kurata M, Manabe S, Koresawa M, Irita J, Enomoto D, Miyoshi K, Fukuoka T, Higaki J. Relationship between cardio-ankle vascular index (CAVI) and carotid atherosclerosis in patients with essential hypertension. Hypertens Res 2007; 30: 335-340.

9 Korkmaz L, Erkan H, Korkmaz AA, Acar Z, Agac MT, Bektas H, Akyuz AR, Adar A, Celik $\mathrm{S}$. [Relationship of aortic knob width with cardio-ankle vascular stiffness index and its value in diagnosis of subclinical atherosclerosis in hypertensive patients: a study on diagnostic accuracy]. Anadolu Kardiyol Derg 2012; 12: 102-106.

10 Căpuşă C, Stancu S, Bădulescu M, Ilyes A, Anghel C, Mircescu G. Determinants of Arterial Stiffness in Chronic Kidney Disease Stages. http://f1000.com/posters/browse/ summary/1687. Accessed 2011.

11 Takaki A, Ogawa H, Wakeyama T, Iwami T, Kimura M, Hadano Y, Matsuda S, Miyazaki Y, Matsuda T, Hiratsuka A, Matsuzaki M. Cardio-ankle vascular index is a new noninvasive parameter of arterial stiffness. Circ J 2007; 71: 1710-1714.

12 Zhou Y, Guo W. Ultrasonic medicine[M]. Science and Technology Literature Press; Beijing, 2002, 796-805.

13 Dengfeng G, Jiang H, Xigui W, Xiufang D, Chonghua Y, Jialiang W, Reynolds K, ChungShiuan C, Klag MJ, Whelton PK. Main causes and its modifiable risks of death among men and women in China. Chin J Prev Chron Noncommun Dis 2006; 14: 149-154.

14 Graham MR, Evans P, Davies B, Baker JS. Arterial pulse wave velocity, inflammatory markers, pathological GH and IGF states, cardiovascular and cerebrovascular disease. Vasc Health Risk Manag 2008; 4: 1361-1371.

15 O'Leary DH, Polak JF, Kronmal RA. Carotid-artery intima and media thickness as a risk factor for myocardial infarction and stroke in older adults. N Engl J Med 1999; 340: 14-22.

16 Wada T, Kodaira K, Fujishiro K. Correlation of ultrasound-measured common carotid artery stiffness with pathological findings. Arterioscler Thromb 1994; 14: 479-482.

17 Yambe T, Yoshizawa M, Saijo Y. Brachio-ankle pulse wave velocity and cardio-ankle vascular index (CAVI). Biomed Pharmacother 2004; 58: 95-98.

18 Bramwell JC, Hill AV. The velocity of the pulse wave in man. Proc $R$ Soc Lond (Biol) 1922; 93: 298-306

19 Hayashi K, Handa H, Nagasawa S, Okumura A, Moritake K. Stiffness and elastic behavior of human intracranial and extracranial arteries. J Biomech 1980; 13: 175-184.

20 Ogawa T, Shimada M, Ishida H, Matsuda N, Fujiu A, Ando Y, Nitta K. Relation of stiffness parameter beta to carotid arteriosclerosis and silent cerebral infarction in patients on chronic hemodialysis. Int Urol Nephrol 2009; 41: 739-745.

21 Hayashi K, Sato M, Niimi H, Handa H, Moritake K. [Analysis of the constitutive laws of the vascular wall by finite deformation theory]. Iyodenshi To Seitai Kogaku 1975; 13: 293-298.

22 Nakamura K, Tomaru T, Yamamura S, Miyashita Y, Shirai K, Noike H. Cardio-ankle vascular index is a candidate predictor of coronary atherosclerosis. Circ J 2008; 72 : 598-604.

23 Kadota K, Takamura N, Aoyagi K, Yamasaki H, Usa T, Nakazato M, Maeda T, Wada M, Nakashima K, Abe K, Takeshima F, Ozono Y. Availability of cardio-ankle vascular index (CAVI) as a screening tool for atherosclerosis. Circ J 2008; 72: 304-308.

24 Soska V, Dobsak P, Dusek L, Shirai K, Jarkovsky J, Novakova M, Brhel P, Stastna J, Fajkusova L, Freiberger T, Yambe T. Cardio-Ankle Vascular Index in Heterozygous Familial Hypercholesterolemia. J Atheroscler Thromb 2012; 19: 453-461.

25 Stamatelopoulos KS, Armeni E, Georgiopoulos G, Kazani M, Kyrkou K, Stellos K, Koliviras A, Alexandrou A, Creatsa M, Papamichael C, Lambrinoudaki I. Recently postmenopausal women have the same prevalence of subclinical carotid atherosclerosis as age and traditional risk factor matched men. Atherosclerosis 2012; 221: 508-513.

26 Namekata T, Suzuki K, Ishizuka N, Shirai K. Establishing baseline criteria of cardioankle vascular index as a new indicator of arteriosclerosis: a cross-sectional study. BMC Cardiovasc Disord 2011; 11: 51

27 Suzuki J, Sakakibara R, Tomaru T, Tateno F, Kishi M, Ogawa E, Kurosu T, Shirai K. Stroke and cardio-ankle vascular stiffness index. J Stroke Cerebrovasc Dis (e-pub ahead of print 19 August 2011). 